\title{
Two Years versus One Year of Tianjiu Therapy in Sanfu Days for Chronic Asthma: A Clinical Efficacy Observation Trial
}

\author{
Li Bing Zhu, ${ }^{1}$ Wei Zhang, ${ }^{1}$ Vivian Wong, ${ }^{1}$ Ziea Eric, ${ }^{2}$ Kwai Ching Lo, \\ Wai Chung Chan, ${ }^{1}$ To Yau, ${ }^{1}$ and Lei Li ${ }^{1}$ \\ ${ }^{1}$ School of Chinese Medicine, The University of Hong Kong, 10 Sassoon Road, Pokfulam, Hong Kong \\ ${ }^{2}$ Chinese Medicine Department, Hospital Authority, Hong Kong
}

Correspondence should be addressed to Lei Li; llie@hku.hk

Received 5 July 2014; Accepted 18 August 2014; Published 11 September 2014

Academic Editor: Jin-Yuarn Lin

Copyright (C) 2014 Li Bing Zhu et al. This is an open access article distributed under the Creative Commons Attribution License, which permits unrestricted use, distribution, and reproduction in any medium, provided the original work is properly cited.

Background. Tianjiu therapy has established efficacy against chronic asthma with related symptoms or the medication need during asthma attack. This study aimed to explore the optimal duration of Tianjiu therapy for asthma. Methods. This study was a selfcomparison-to-the-baseline study, which comparing treatment with Tianjiu therapy for 1 year and 2 years in the same 102 chronic asthma patients. Totally 6 sessions of Tianjiu treatment were provided, 3 sessions in a year as a course of treatment and totally two years treatment. The primary endpoint was the number of asthma related symptoms which frequently appeared in asthma patients and the frequency of bronchodilator used during asthma attack. Results. The frequency of bronchodilator used during asthma attack significantly improved $\left(\chi^{2}=46.276, P=0.000\right)$. But the number of asthma related symptoms which frequently appeared in asthma patients added by 1.38 points (95\% CI, 0.25 to 2.51$), 2.93 \pm 0.41$ in 1-year group and $4.31 \pm 0.41$ in the 2 -years group $(P<0.05)$. Conclusions. The effect of 2 years Tianjiu therapy was not as effective as 1 year such treatment for asthma, but the second year Tianjiu therapy was still needed because it has a role to consolidate the curative effect of Tianjiu therapy for asthma.

\section{Introduction}

Asthma is one of the most common chronic diseases in the world. Currently, $\beta$-agonist as the first-line drug in treating acute asthma attack and glucocorticoids is still used widely to treat chronic asthma [1]. From the perspective of immunology, asthma is a systemic allergic disease with immune disturbance. Allergic airway inflammation is only a local show, and inhaled glucocorticoids also only pay attention to the local anti-inflammatory therapy while overlooking the systemic immune dysfunction in asthma patients. Hence, current therapy for asthma is still imperfect; it needs us to keep improving the therapy for asthma, and immunotherapy is likely to be one of the important ways to improve asthma therapy [2]. Traditional Chinese medicine (TCM) has a long history of treating asthma and it claimed that the mechanism of asthma mainly lies in two aspects: one is "deficiency in origin" and another is "enrichment in symptom." The saying of "deficiency in origin" is similar to "systemic immune dysfunction," and "enrichment in symptom" is similar to "allergic airway inflammation." TCM pays more attention to the "preventive treatment of disease," which means conduct prevention treatment for asthma patients when there is no asthma attack. Tianjiu Therapy in Sanfu Days is a classic prevention treatment for asthma. Sanfu Days means the three hottest days in a year which are calculated by ancient calendar. Both Positive-qi in human body and nature are in a most exuberant status in Sanfu Days, so Sanfu Days is a good time for cold-insufficiency patients to tonic Positive-qi. As a result, patients can have a strong body-resistance to against exogenous pathogen because they have already accumulative enough Positive-qi inside. Just as Chinese medicine said: "when there is sufficient Positive-qi inside, the Pathogenicqi has no way to invade the health body." Tianjiu Therapy means applying herbs patches on special acupoints in order to stimulate skin to form blisters, hyperemia, and even suppuration. As a result, Sanfu Tianjiu Therapy can attain the goal of strengthening Yang-qi, removing cold pathogen, 
and enhancing body resistance through the combination effect of drug infiltration absorption, acupoints stimulation, and time effect. Sanfu Tianjiu Therapy for asthma aims to improve the body immunity which in turn can get a purpose of preventing and reducing respiratory viral or bacterial infection, reducing airway inflammation injury, and reducing airway hyperresponsiveness which in turn reduces the times of asthma attacks [3-12]. Tianjiu Therapy substantially improves asthma control level and the quality of life in patients with asthma and is used widely in Mainland China as an adjuvant setting [3-12]. We have already conducted an initial trial which compared 1 year of Tianjiu treatment with a placebo control group. In that study, it was found that Tianjiu Therapy can really reduce the need for medications to control asthma, improved the quality of participants' life, and significantly reduced the level of asthma. Previous study has preliminary confirmed the effect of Tianjiu Therapy in Sanfu Days for asthma, while the optimal treatment duration is still unknown. Hence this study was conducted to compare the different effect of 2-year Tianjiu Therapy with 1-year such treatment in asthma patients.

\section{Methods}

2.1. Enrollment Criteria. This study was a self-control and clinical efficacy observation trial in 102 Hong Kong citizens (above 13 years of age) with chronic asthma. Participants need to provide physician's diagnosis of asthma or documentation of asthma-related symptoms preceding study visit. Besides, all participants should provide evidence of asthma attack as indicated by hospitalization or unscheduled urgent care twelve months before study entry. In addition, all patients were required to meet the following inclusion criteria: cough (worse at night), difficulty in breathing, chest tightness, awakening the patient, and symptoms occurring or worsening with exercise or at night; episodic symptoms of airflow obstruction, viral infections, strong emotions, and changes in weather. Patients would be assessed ineligibility according to one or more of the following exclusion criteria: severe cardiac and pulmonary diseases; fever, pharyngitis, acute asthma attack, and pregnancy. Diabetes mellitus, tuberculosis, and hypersensitive skin condition were also forming the excluded reasons. Additionally, allergy to topical medication, severe heart diseases, keloid, bleeding disorders or participants with pacemaker will also be excluded.

This study was approved by the Institutional Review Board of the University of Hong Kong/Hospital Authority Hong Kong West Cluster (HKU/HA HKW IRB) with coding UW 13-316. All participants were informed about this study and written informed consent was obtained from every patient or patient's legal guardian or the patient's parent.

2.2. Study Design. In this study, participants from 2010 to 2013 who totally receive two courses of Tianjiu Therapy (one course means completing three-time Tianjiu Therapy in a year, so two-course treatment needs at least two years and sixtime Tianjiu Therapy) will constitute study subjects for final

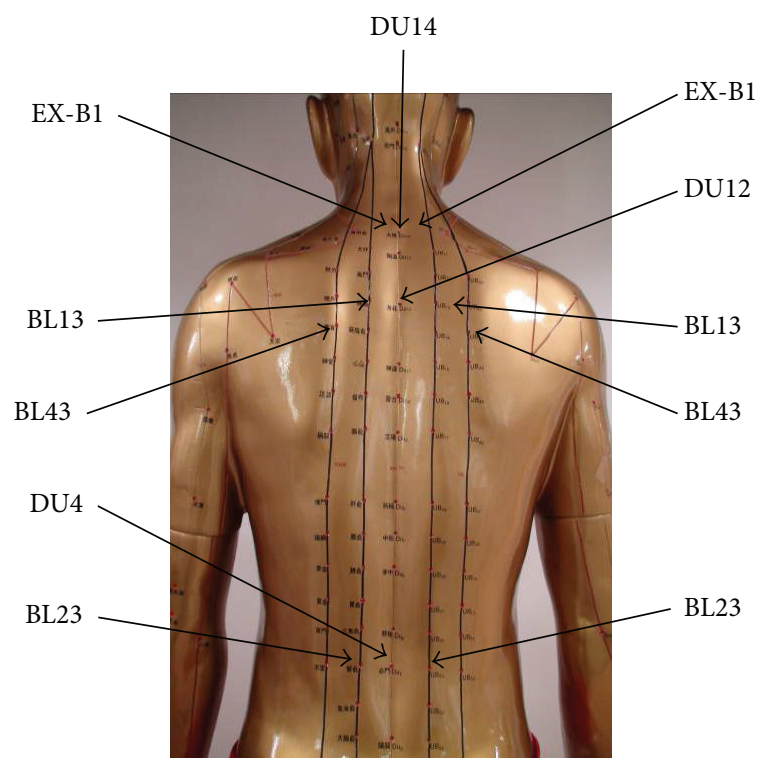

Figure 1: Acupoints.

analysis. For example, patients who have already received 1course Tianjiu Therapy in 2010 need to receive another course treatment in 2011 or 2012 or 2013. Patients from placebo group in 2010 still need to receive other 2-course treatment in the following three years. As a result, 102 patients who totally received two-course treatment were included for the final analysis. These 102 patients must complete two-course preassessment and postassessment which means that they have completed data in four time points. Among these 102 patients, there were 68 continuous participants (attended in 2010 and 2011, 2011 and 2012, or 2012 and 2013) plus 34 discontinuous participants (attended in 2010 and 2012, 2010 and 2013, or 2011 and 2013). This study conducted a subgroup analysis for continuous group and discontinuous group, but nothing different was found in most of the outcome measurements. Hence, this study combined continuous participants with discontinuous participants as study subjects.

Each year Asthma Control Test (ACT), self-made questionnaire, and pulmonary function will be assessed for participants before treatment and after treatment. Also threetime Tianjiu Therapy with 2-hour duration in a year was conducted for all participants from 2010 to 2013 and every time Tianjiu Therapy was conducted in Sanfu Days. In other words, eligibility patients participated in a certain year study then that year's three treatments and pre and post assessment were conducted for them. The three treatment times from 2010 to 2013 were July 19, July 29, and August 8, 2010; July 14, July 24, and August 13, 2011; July 18, July 28, and August 7, 2012; July 13, July 23, and August 12, 2013.

Eleven acupoints will be applied by Tianjiu patches: BL13 (both sides), BL23 (both sides), BL43 (both sides), EX-B1 (both sides), Du14, Du12, and Du4 (Figure 1).

One patch was applied on one acupoint and every patch weighted 2 grams (Figure 2).

The formula of Tianjiu patch was a combination of the formula frequency used in clinical trials and the record in 


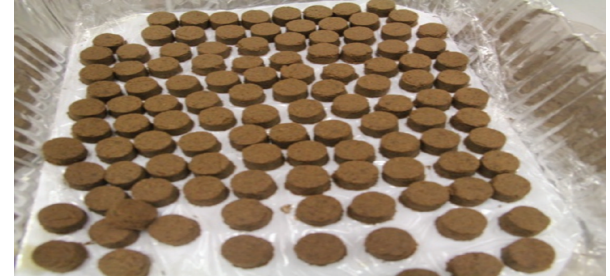

Figure 2: Tianjiu patch.

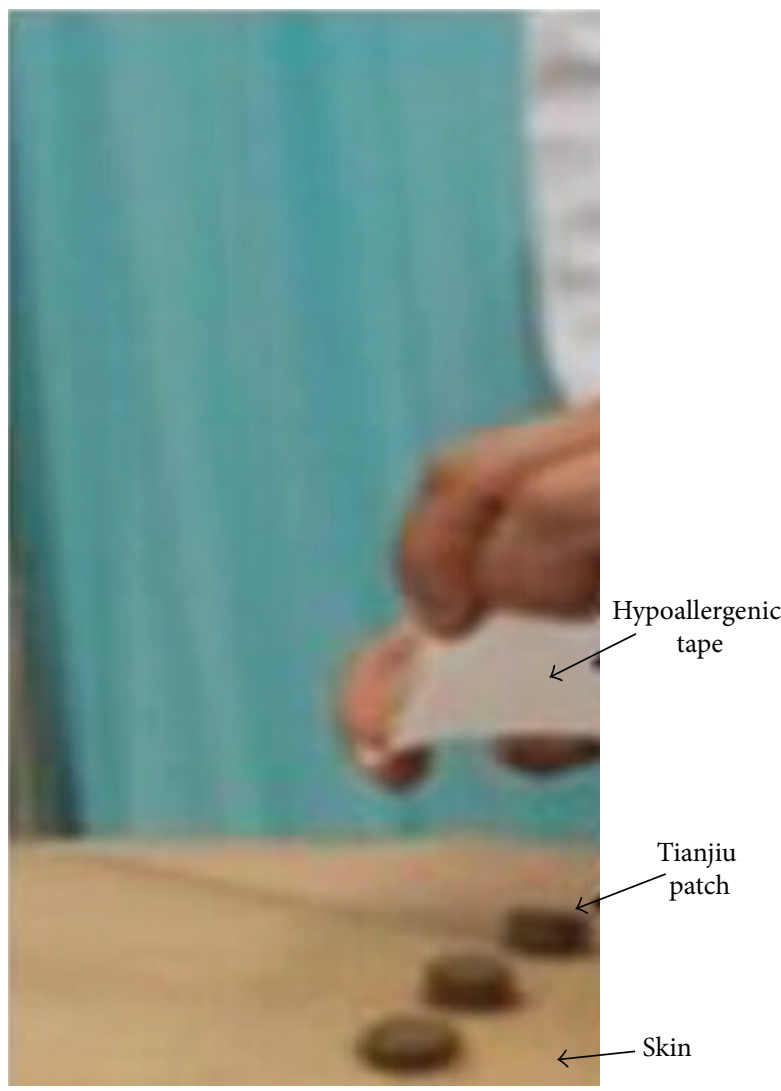

FIgURE 3: Tianjiu Therapy schematic diagram.

"Zhang Shi Yi Tong." The final formula used consisted of Sinapis alba, Radix Corydalis Yanhusuo, processed Euphorbia kansui, Asari Herba cum Radice, Ephedrae Herba, processed Radix Aconiti Praeparata, Cinnamomum cassia, and Eugenia caryophyllata. The ratio of selected herbal was $2: 1: 1: 1: 1: 1: 1: 1$ and all this herbal powder will be mixed together with ginger juice. Finally, approximately $4 \mathrm{~cm} \times 4 \mathrm{~cm}$ hypoallergenic tape (3M Micropore Tape 1535-3) will be used to stick Tianjiu patches on skin after the patch was applying on acupoints (Figure 3).

2.3. Outcome Measures. The primary outcome was the number of asthma-related symptoms which frequently appeared in asthma patients and the frequency of bronchodilator used during asthma attack. Other outcomes included the number of days with asthma-related symptoms in 1 month before visit; spirometric measurements; asthma-related health care use and the score on the ACT. Scores on the ACT range from 5 to 25 , with scores of 20 or more indicating disease control and 3 points are the minimally important difference for the ACT score [13].

2.4. Statistical Analysis. Continuous variables in this study from 1st year baseline to 2nd year posttreatment were examined using a linear mixed-effects model with time as fixed effects. There were totally four time points in this linear mixed-effects model: 1st year baseline as the 1st time point, 1 st year posttreatment as the 2 nd time point, 2 nd year baseline as the $3 \mathrm{rd}$ time point, and 2 nd year posttreatment as the 4 th time point. The continuous variables included the score of ACT, the number of asthma-related subhealthy symptoms, the number of days with asthma-related symptoms, and lung function: FEV and $\mathrm{FEV}_{1} / \mathrm{FVC}(\%)$. All analysis was conducted for the same 102 people. Generalized estimated equation (GEE) analysis was performed for the frequency of bronchodilator used during asthma attack and asthmarelated health care used during asthma attack in order to repeat measures in different time points. Descriptive analysis was conducted for the percentage of patients with twentythree asthma-related symptoms which frequently appeared in asthma patients. Statistical analyses were performed with the Statistical Package for the Social Sciences (SPSS) software program (version 19) for windows XP.

\section{Results}

From June 2010 through July 2010, 323 patients were recruited in 2010 and finally 242 patients completed treatment. 274 patients were recruited as study subjects in 2011, of which 84 participants were from 2010 Tianjiu Therapy group, 82 were from 2010 placebo group, and 108 were new recruited subjects. During the treatment period and postassessment in 2011, 104 patients did not complete all parts. As a result, 170 participants completed all treatments and postassessment in 2011. Totally 132 patients completed all treatments and pre- and postassessments in 2012, and 150 patients lastly completed preassessments, postassessments, and three-time Tianjiu Therapy in 2013. Patients would be ineligible for final statistical analysis due to the incomplete data. The cause of incomplete data included incomplete treatments, during treatment period participants losing contact, illnesses, pregnancy, asthma exacerbation, inability to participate timely, refusing to participate, or incomplete questionnaires, ACT, or lung function test. The patients can be included for final analysis only when they have four time points' data (1st year preand postassessments, 2nd year pre- and postassessments). Overall, from 2010 to 2013, patients who received two-course treatment were 102.

For the baseline characteristics of these 102 participants, the average age was 50.4 years (interquartile range, 13 to 78); $53 \%$ were female (Table 1 ). The average number of days on which patients had asthma-related symptoms was 9.35. Patients had a mean ACT score of 19 or less which indicated a lack of disease control [14]. In the previous year, $22.5 \%$ patients received emergency treatments at least once 
TABLE 1: Baseline characteristics of study participants.

\begin{tabular}{|c|c|}
\hline Characteristic & $\begin{array}{l}\text { Tianjiu Therapy } \\
\qquad(N=102)\end{array}$ \\
\hline Age-year & $50.35 \pm 17.17$ \\
\hline \multicolumn{2}{|l|}{ Gender-number (\%) } \\
\hline Male & $48(47.1)$ \\
\hline Female & $54(52.9)$ \\
\hline Duration of asthma-year & $20.22 \pm 14.14$ \\
\hline Asthma-related symptoms-number of ${ }^{\mathrm{a}}$ & $9.35 \pm 11.90$ \\
\hline \multicolumn{2}{|l|}{ Lung function } \\
\hline $\mathrm{FEV}_{1}$ & $1.86 \pm 0.98$ \\
\hline $\mathrm{FEV}_{1}: \mathrm{FVC} \times 100$ & $85.24 \pm 27.76$ \\
\hline \multicolumn{2}{|l|}{ Asthma-related health care-number (\%) } \\
\hline$\geq 1$ Admitted to $\mathrm{A}$ and $\mathrm{E}^{\mathrm{b}}$ & $23(22.5)$ \\
\hline$\geq 1$ Hospitalization & $19(18.6)$ \\
\hline$\geq 1$ Outpatient visit & $60(58.8)$ \\
\hline$\geq 1$ Prescription & $72(70.6)$ \\
\hline$\geq 1$ Buy medication & $11(10.8)$ \\
\hline$\geq 1$ Not to be processed & $10(9.8)$ \\
\hline \multicolumn{2}{|l|}{$\begin{array}{l}\text { The frequency of bronchodilator used } \\
\text { during asthma attack-number (\%) }\end{array}$} \\
\hline More than twice per day & $25(24.5)$ \\
\hline Once to twice per day & $38(37.3)$ \\
\hline 2-3 times per week & $5(4.9)$ \\
\hline Less than once per week & $16(15.7)$ \\
\hline Never & $18(17.6)$ \\
\hline $\begin{array}{l}\text { Symptoms which frequently appeared in } \\
\text { asthma patients-number of symptoms }{ }^{c}\end{array}$ & $8.20 \pm 4.40$ \\
\hline
\end{tabular}

${ }^{a}$ Plus-minus values are means $\pm \mathrm{SD}$, unless noted otherwise. PIF denotes peak inspiratory flow; PEF: peak expiratory flow; $\mathrm{FEV}_{1}$ : forced expiratory volume in one second; FVC: forced vital capacity.

${ }^{\mathrm{b}}$ The total number of symptoms which frequently appeared in asthma patients is 23 and its scale ranges from 0 to 23 .

${ }^{c}$ Accident and Emergency Departments (A and E).

which were associated with an asthma-related event, $18.6 \%$ had been hospitalized, $58.8 \%$ went to clinic, $70.6 \%$ treated themselves following the prescription, $10.8 \%$ buy medication for themselves, and 9.8\% did not need medical treatment. The mean $( \pm \mathrm{SD}) \mathrm{FEV}_{1}$ was $1.86 \pm 0.98$, and the mean ration of $\mathrm{FEV}_{1}$ to the forced vital capacity (FVC) was $85.24 \pm 27.76$. For the frequency of bronchodilator used during asthma attack, $24.5 \%$ used bronchodilator more than twice per day, $37.3 \%$ once to twice per day, $4.9 \% 2-3$ times per week, and $15.7 \%$ less than once per week, and $17.6 \%$ never used bronchodilator during asthma attack. The number of symptoms which frequently appeared in asthma patients was $8.20 \pm 4.40$.

3.1. Response to Intervention. The number of symptoms which frequently appeared in asthma patients was $2.93 \pm 0.41$ in end of 1-course treatment and $4.31 \pm 0.41$ in end of 2 course treatment; one course has 5.27-point reduction and 2 courses have 3.88-point reduction, respectively, comparing with $8.20 \pm 0.41$ in baseline $(P<0.05)$ (Figure 4 and Table 2$)$.

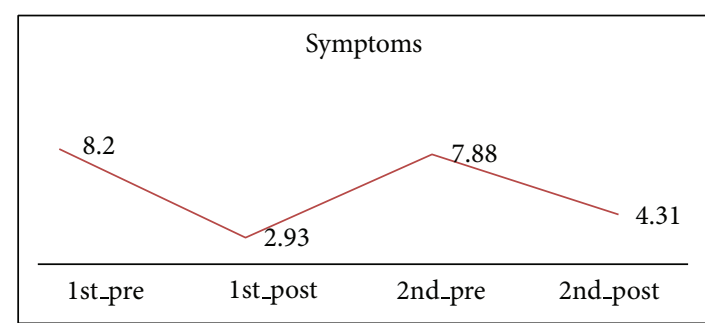

- Symptoms

FIgURE 4: The number of symptoms which frequently appeared in asthma patients in 1 course and 2 courses.

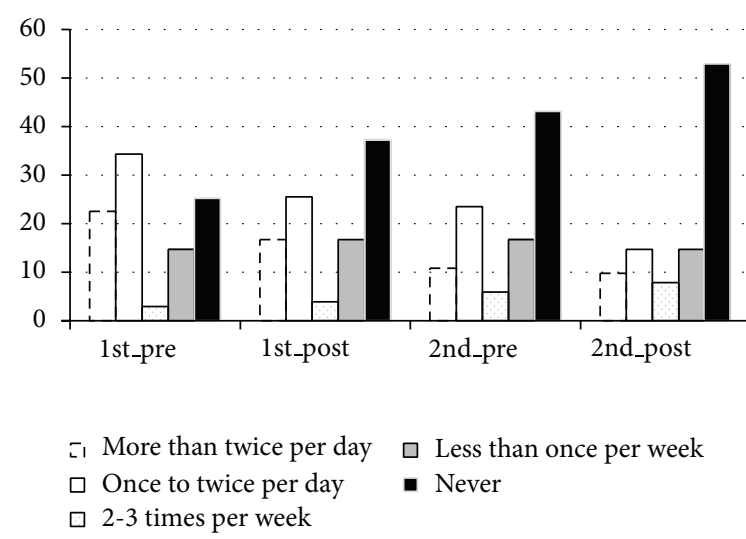

FIGURE 5: The frequency of bronchodilator used during asthma attack.

But 1-course treatment reduced more points than 2-course treatment $(P<0.05)$.

The frequency of bronchodilator used during asthma attack in 2 courses was better than 1 course: $16.7 \%$ more than twice per day in 1 course and $9.8 \%$ in 2 courses, $25.5 \%$ once to twice per day in 1 course and $14.7 \%$ in 2 courses, $3.9 \% 2$ to 3 times per week in 1 course and $7.8 \%$ in 2 courses, and $16.7 \%$ less than once per week in 1 course and $14.7 \%$ in 2 courses, and $37.3 \%$ never used it in 1 course and $52.9 \%$ in 2 courses, indicating that the frequency of bronchodilator used during asthma attack was decreasing (Table 3 and Figure 5, $P<0.05$ ).

The score of ACT improved by 1.53 points ( $95 \%$ CI, -0.31 to 3.37) after one-course treatment (19.80) and 3.53 points (95\% CI, 1.69 to 5.37 ) after two courses of treatment (21.80) (Figure 6 and Table 4). In other words, the score of ACT after two-course treatment was better than one-course treatment and the score of ACT showed an increasing trend $(P<0.05)$.

Different from the improvement like the score of ACT and the frequency of bronchodilator used during asthma attack, as compared with 1-year treatment, 2-year treatment with Tianjiu increased the mean number of days on which patients had asthma-related symptoms from 1.98 to $5.03(P<$ 0.05 ) (Table 2 and Figure 7); even both 2-year treatment and 1-year treatment compared with baseline have a significant improvement $(P<0.05)$.

No changes occurred in pulmonary function for both 1-year treatment and 2-year treatment comparing with no 


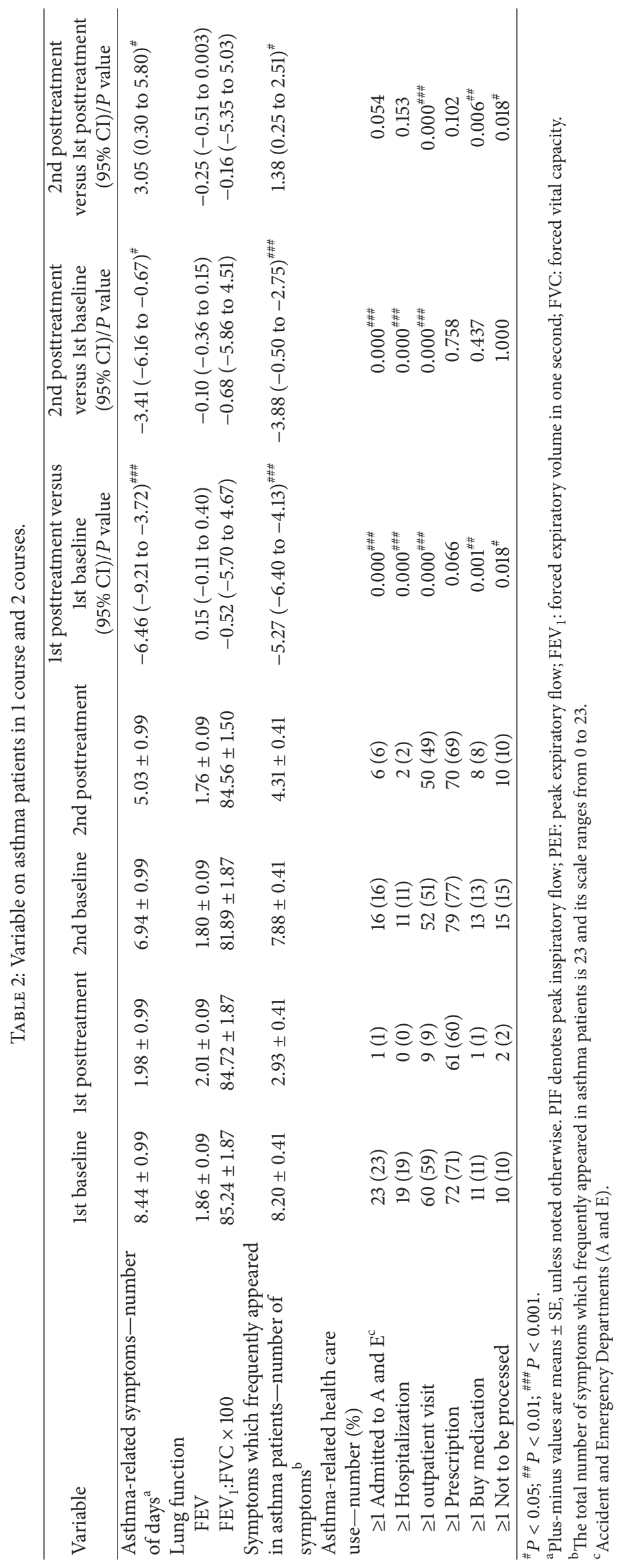


TABLE 3: The frequency of bronchodilator used during asthma attack $-n(\%)$.

\begin{tabular}{|c|c|c|c|c|}
\hline \multirow{2}{*}{ Variable } & \multicolumn{4}{|c|}{ Time } \\
\hline & 1st_pre & 1st_post & 2nd_pre & 2nd_post \\
\hline More than twice per day & $23(22.5)$ & $17(16.7)$ & $11(10.8)$ & $10(9.8)$ \\
\hline Once to twice per day & $35(34.3)$ & $26(25.5)$ & $24(23.5)$ & $15(14.7)$ \\
\hline 2-3 times per week & $3(2.9)$ & $4(3.9)$ & $6(5.9)$ & $8(7.8)$ \\
\hline Less than once per week & $15(14.7)$ & $17(16.7)$ & $17(16.7)$ & $15(14.7)$ \\
\hline Never & $26(25.2)$ & $38(37.3)$ & $44(43.1)$ & $54(52.9)$ \\
\hline
\end{tabular}

Time main effect: Wald $\chi^{2}=46.276, P=0.000$.

TABLE 4: ACT score before and after treatment in 1 course and 2 courses.

\begin{tabular}{lc}
\hline 1st baseline & $18.27 \pm 0.66$ \\
1st posttreatment & $19.80 \pm 0.66$ \\
2nd baseline & $20.43 \pm 0.66$ \\
2nd posttreatment & $21.80 \pm 0.66$ \\
1st posttreatment versus 1st baseline & $1.53(-0.31$ to 3.37$)$ \\
$\begin{array}{l}\text { (95\% CI }) / P \text { value } \\
\text { 2nd posttreatment versus 1st baseline }\end{array}$ & $3.53(1.69 \text { to } 5.37)^{\# \# \#}$ \\
$\begin{array}{l}\text { (95\% CI }) / P \text { value } \\
\text { 2nd posttreatment versus 1st } \\
\text { posttreatment }(95 \% \mathrm{CI}) / P \text { value }\end{array}$ & $2.00(0.16 \text { to } 3.84)^{\#}$ \\
\hline
\end{tabular}

Notes: ${ }^{\#} P<0.05 ;{ }^{\# \#} P<0.001$.

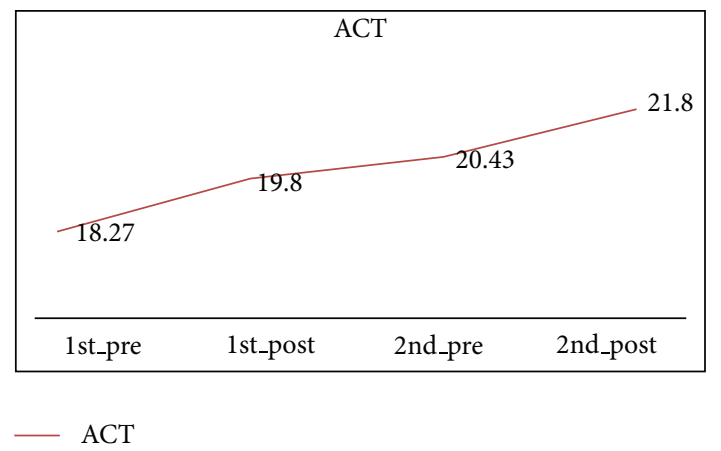

FIGURE 6: The score of ACT in 1 course and 2 courses.

treatment status. With respect to asthma-related health care use during asthma attack, the percentage of participants admitted to Accident and Emergency Departments ( $\mathrm{A}$ and E) was $1 \%$ in year 1 and $6 \%$ in 2 years; comparing with baseline both have a significant improvement $(P<0.05)$. Similarly, the proportion of patients who were hospitalized due to asthma was $0 \%$ in 1 year and $2 \%$ in 2 years, with both being better than no treatment $(P<0.05)$. The percentage of participants who visited outpatient clinic was $9 \%$ in 1 year and $49 \%$ in 2 years; both of them have a significant improvement in comparison with no treatment; however, the effect of 1 year was much better than 2 years $(P<0.05)$. The ratio of subjects who took the previous prescription by doctors during asthma attack was $60 \%$ in 1 year and $69 \%$ in 2 years, and no difference occurred in both groups comparing with no treatment $(P>0.05)$; the scale of patients who only need

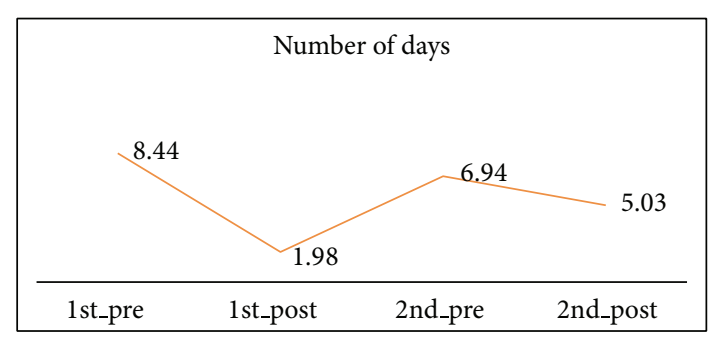

_ Number of days

FIGURE 7: The number of days with asthma-related symptoms in 1 year and 2 years.

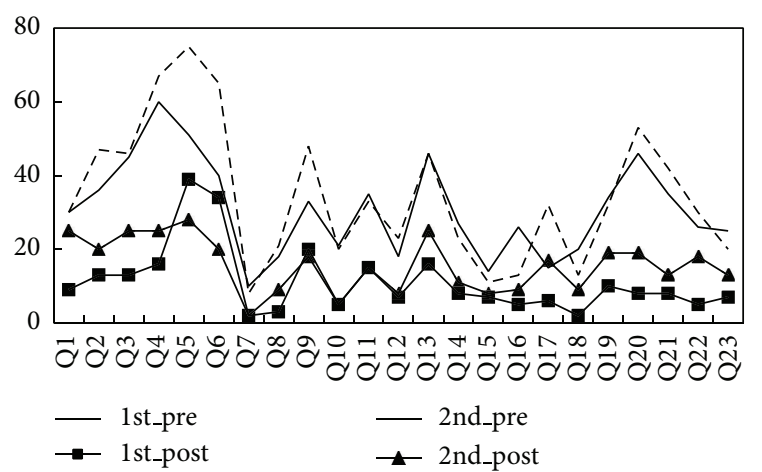

FIGURE 8: The proportion of participants with twenty-three symptoms which frequently appeared in asthma patients in 1 course and 2 courses.

to take medication by themselves was $1 \%$ in 1 year and $8 \%$ in 2 years; 1-year treatment was better than no treatment while 2year treatment was not different from baseline, indicating that 1 -year treatment was better than 2-year treatment $(P<0.05)$; last but not least, the percentage of patients who do not need to process during asthma attack was $2 \%$ in 1 year and $10 \%$ in 2 years; 2 -year treatment has nothing different from baseline, while 1-year treatment was worse than no treatment and 2 years performed better than 1 year $(P<0.05)$ (Table 2$)$.

Figure 8 showed the percentage of participants with twenty-three symptoms which frequently appeared in asthma patients. Among these twenty-three symptoms, the top three frequently occurring symptoms were as follows: easy onset during quarter turn (67\% in baseline, $16 \%$ after 1-course treatment, and $25 \%$ after 2 -course treatment), rapid or difficult 
breathing ( $75 \%$ in baseline, 39\% after 1-course treatment, and $28 \%$ after 2 -course treatment), and waking up with asthma symptoms (65\% in baseline, $34 \%$ after 1-course treatment, and $20 \%$ after 2-course treatment), indicating that the majority of participants owned the lung-qi-deficiency syndromes. Nearly all patients have a significant reduction in the twenty-three symptoms after both 1-course Tianjiu Therapy and 2-course such treatment. What is more, it showed that the status of patients in the 2nd course baseline was better than 1st course baseline which indicated that Tianjiu Therapy has a cumulative effect after one course of treatment. Additionally, from Figure 8, it can be found that the reduction of 1 course was much more than 2 courses. It seems that the effect of 2 courses was not as sensitive as 1 course, regardless of the fact that 2-course treatment still has a significant improvement compared with 1st course baseline.

3.2. Adverse Event. The observation procedures followed after Tianjiu Therapy were conducted in both 1 course and 2 courses. During the treatment, most patients would show cutaneous reaction such as skin warm feeling, itching, pain, and blisters due to the stimulation of herbal patch. All these appearances were a normal skin reaction after Tianjiu Therapy and also a key condition of Tianjiu Therapy taking effect in the treatment of asthma. Most of them would disappear after several hours or several days. In 1st course Tianjiu Therapy, there were five serious adverse events reported at the end of three-time treatment: three patients showed asthma exacerbation, one patient showed the rash due to being allergic to Tianjiu patch, and one subject tended to vomit blood and automatically stopped after the 2nd Tianjiu Therapy, which had confirmed that it has nothing to do with Tianjiu Therapy. Besides, in 2nd course Tianjiu Therapy, the majority of patients also showed skin reaction after Tianjiu Therapy as that in 1st course. There were also five serious adverse events in the 2nd course treatment: three were skin sensibility which cannot disappear after stopping treatment even several days later. And finally, his skin returned to normal through dermatologist's appropriate process. one person was caused by the smell of paint because his house was mopping during the treatment period; another was induced by flu. Overall, there was nothing different between 1st course Tianjiu Therapy and 2nd course Tianjiu Therapy in adverse events.

\section{Discussion}

In the previous study (HKCTR-1128), Tianjiu Therapy has shown substantial improvements in the number of days with asthma-related symptoms, the number of symptoms which frequently appeared in asthma patients, and the medication need after treatment. Although there was nothing different between placebo group and Tianjiu Therapy group after the 3rd treatment immediately, the effect of Tianjiu Therapy tended to be superior to placebo group in four-time followup. This study was conducted to explore how long the curative effect of Tianjiu Therapy lasts and the optimum duration of treatment for chronic asthma based on previous finding. This study tested the hypothesis that continuation of Tianjiu
Therapy beyond 1 course would be more effective than 1 course of such treatment. As a result, this study found that two courses of Tianjiu Therapy showed a significant improvement in the score of ACT as compared with 1course treatment and the score of ACT showed an increasing trend. In addition, the frequency of bronchodilator used during asthma attack in 2 courses was also superior to the effect of 1 course. Patients' asthma control situation in 2nd pretreatment was better than that in the 1st pretreatment. Which means patients' immunity improved and kept well after one-course Tianjiu Therapy? While, it was arbitrary to make conclusion that the effect of 2-course Tianjiu Therapy was superior to 1 course by only judging from the change of the score of ACT and the bronchodilator used during asthma attack between 1 course and 2 courses. Nevertheless, different from the findings above, the result of the mean number of days on which patients had asthma-related symptoms shows no such additional benefit; the effect of 2-course treatment was close to 1 course such treatment. Likewise, results about the number of symptoms which frequently appeared in asthma patients, asthma-related health care use during asthma attack, and the percentage of participants with symptoms which frequently appeared in asthma patients were not mostly different between the 1-course treatment and 2-course such treatment. Last but not least, no changes occurred in pulmonary function for both 1-course treatment and 2-course treatment comparing with 1st course baseline.

Although the result was inconsistent in all outcome measurements, it was found that the effect of 2-course Tianjiu Therapy was less sensitive than 1 course of such treatment for chronic asthma, but both 1-course and 2-course Tianjiu Therapy can get a significant improvement comparing with the situation in 1st pretreatment. And the baseline status of the same people in the 2nd course remained well even one year later after the first course of treatment, indicating that the effect of Tianjiu Therapy can last for a quite long time. For safety issue, the number of adverse events in 2nd course treatment was nearly the same as the number in 1st course treatment. There is no doubt that all these findings will be beneficial for health policy makers and asthma patients.

There is a limitation of this study because this study did not set up followups and only takes the second course's baseline as the followup of first course treatment. However, Sanfu Days in every year were around July and August which was a stable period for asthma patients, so it was easy to produce bias without followup in asthma high incidence and research staff cannot track the asthma control situation of participants. Hence, it is urgent and necessary to add followups. Table 5 showed the temperature record of 2010 to 2013. The average temperature in Sanfu Days was $28.7^{\circ} \mathrm{C}$ from 2010 to 2013; the average humidity was $81 \%$ from 2010 to 2013; the average duration of sun exposure was 6.6 hours from 2010 to 2013 .

\section{Conclusion}

Both 2-course Tianjiu Therapy and one-course Tianjiu Therapy significantly reduced the number of symptoms which 


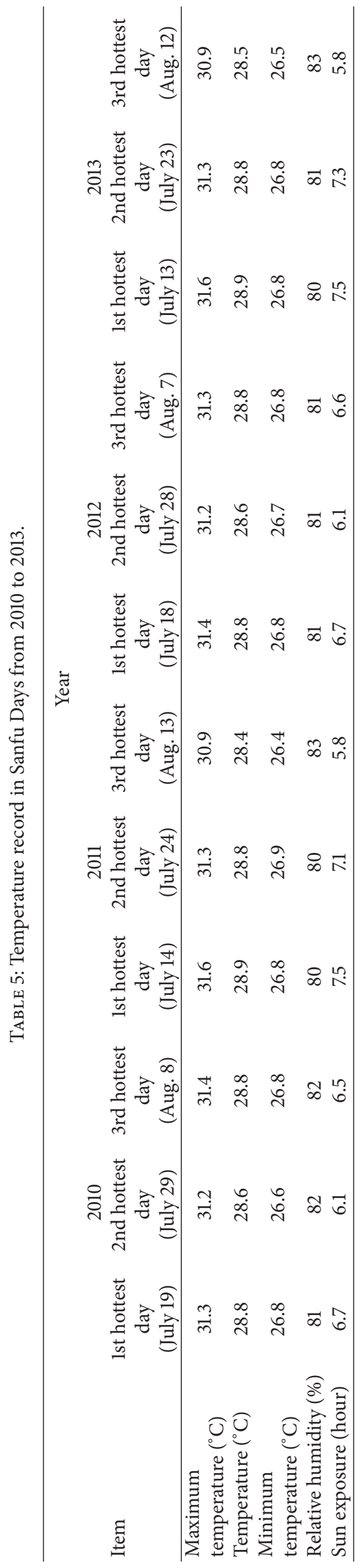


frequently appeared in asthma patients and medication need in participants with chronic asthma as compared with baseline. The asthma control situation in 2 nd course pretreatment was better than 1st course pretreatment. Although the second course Tianjiu Therapy was less sensitive than first course Tianjiu Therapy for chronic asthma, the second course treatment still plays a role in consolidating the curative effect of Tianjiu Therapy in the treatment of asthma.

\section{Conflict of Interests}

The authors declare that there is no conflict of interests regarding the publication of this paper.

\section{References}

[1] W. W. Busse, W. J. Morgan, P. J. Gergen et al., "Randomized trial of omalizumab (anti-IgE) for asthma in inner-city children," The New England Journal of Medicine, vol. 364, no. 11, pp. 1005-1015, 2011.

[2] M. H. Li, K. S. Yin, and Y. Y. Cai, Asthmology, People's Medical Publishing House, Beijing, China, 2005.

[3] E. X. Zeng and Y. J. Chen, "Effect observations of natural moxibustion therapy in bronchus asthma," Modern Nursing, vol. 12 , no. 5, article 412, 2006.

[4] X. S. Lai, Y. M. Li, and J. W. Zhang, "The influence of Tianjiu therapy to sIL-2R and T-cell of asthma patients," Chinese Acupuncture \& Moxibustion, vol. 1, pp. 22-25, 2000.

[5] W. J. Dai and X. S. Lai, "The effect of medicinal vesiculation therapy on plasma SP and VIP contents in bronchial asthma patients," Acupuncture Research, vol. 26, no. 2, pp. 124-127, 2001.

[6] W. Z. Lu, "The impact of acupoint application on cytokines IL-4, IL-10 and IL-12 in asthma patients," Liaoning Journal of Traditional Chinese Medicine, vol. 24, no. 2, pp. 129-120, 2007.

[7] Y. Meng and S. X. Cai, "IL-12 and its role in the mechanism of asthma attack," Journal of First Military Medical University, vol. 9, p. 711, 2001.

[8] J. L. Zhang, "The polymorphisms of IL-4 and IL-10 gene promoter region and asthma," Foreign Medical Sciences-Immunology, vol. 2, article 104, 2002.

[9] J. J. Yang and X. S. Lai, "Research on the efficacy and mechanism of Tianjiu Therapy for bronchial asthma," Jilin Journal of Traditional Chinese Medicine, vol. 22, no. 4, pp. 7-8, 2002.

[10] M. Chen, X. L. Lu, O. R. Zhen et al., "Research on the relationship between the efficacy of Sanfu Tianjiu Therapy and ET, IgE and Lung function," Traditional Chinese Medicine Research, vol. 18, pp. 44-46, 2005.

[11] T. Nagase, Y. Fukuchi, C. Jo et al., "Endothelin-1 stimulates arachidonate 15-lipoxygenase activity and oxygen radical formation in the rat distal lung," Biochemical and Biophysical Research Communications, vol. 168, no. 2, pp. 485-489, 1990.

[12] J. Du, D. Cui, and Y. Guo, "Detection of ET-1, TNF-alpha, oxidative radicals in sera of asthmatics and in sera and lung tissue of asthmatic guinea pigs," Chinese Journal of Tuberculosis and Respiratory Diseases, vol. 21, no. 5, pp. 292-295, 1998.

[13] M. Schatz, M. Kosinski, A. S. Yarlas, J. Hanlon, M. E. Watson, and P. Jhingran, "The minimally important difference of the
Asthma Control Test," Journal of Allergy and Clinical Immunology, vol. 124, no. 4, pp. 719-723, 2009.

[14] M. Schatz, C. A. Sorkness, J. T. Li et al., "Asthma control test: reliability, validity, and responsiveness in patients not previously followed by asthma specialists," Journal of Allergy and Clinical Immunology, vol. 117, no. 3, pp. 549-556, 2006. 


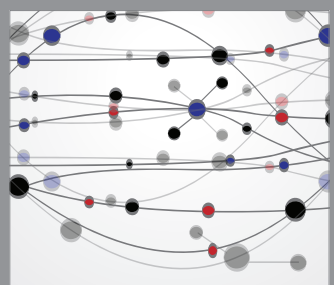

The Scientific World Journal
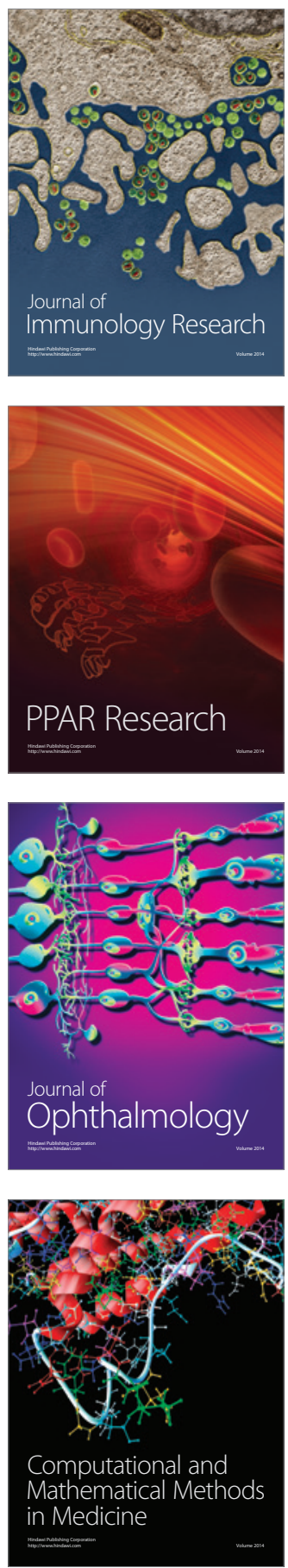

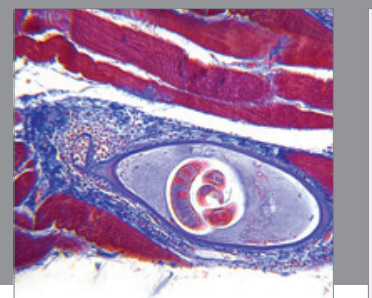

Gastroenterology

Research and Practice
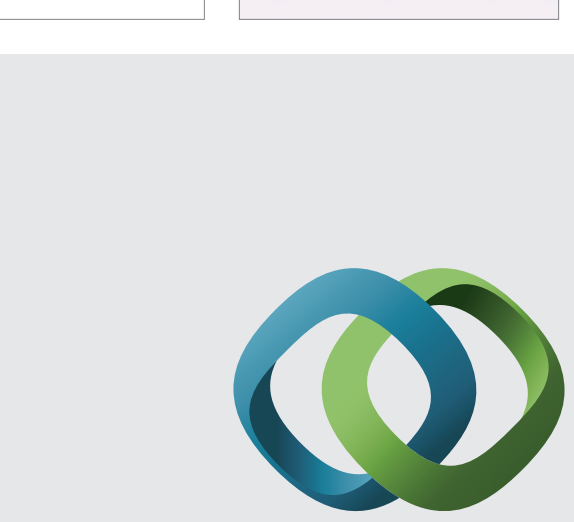

\section{Hindawi}

Submit your manuscripts at

http://www.hindawi.com
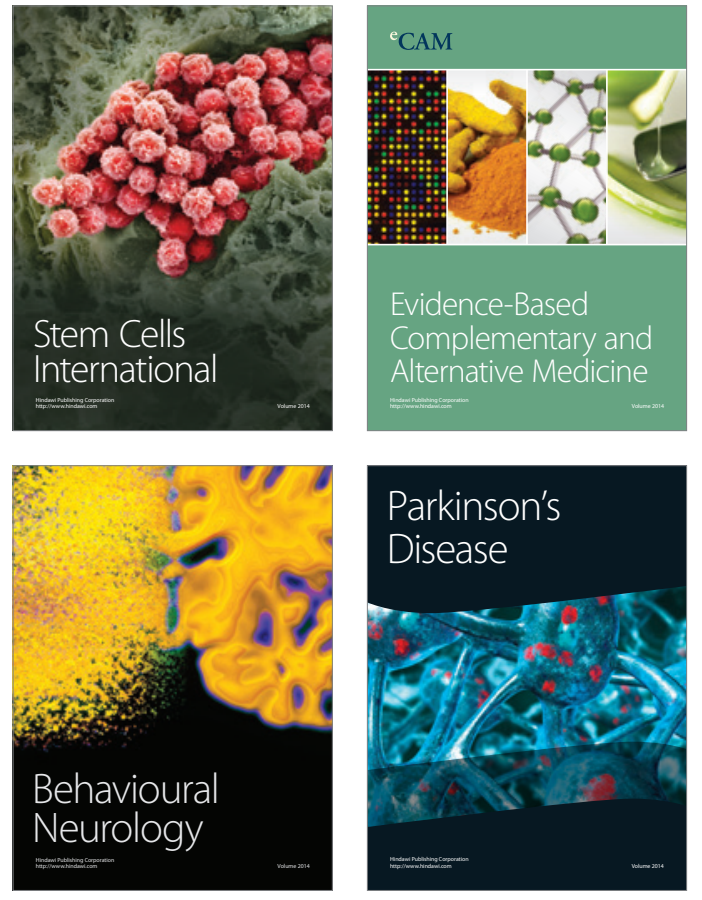
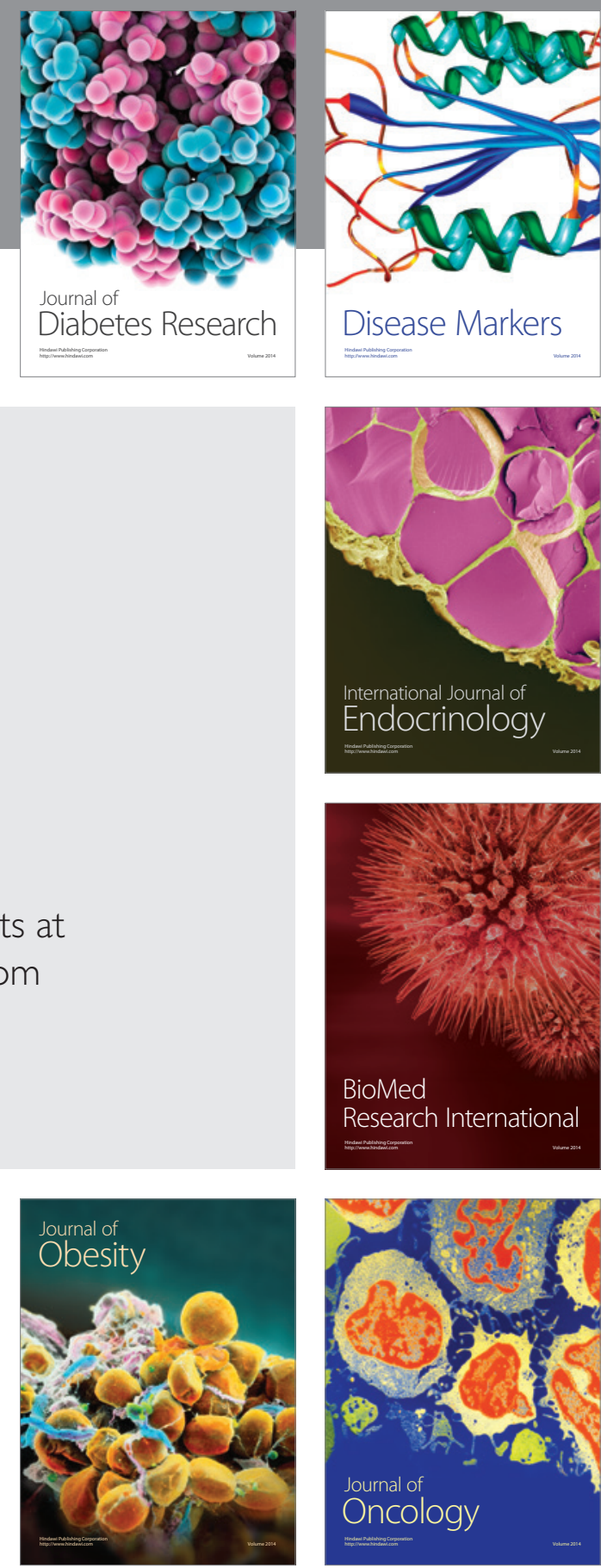

Disease Markers
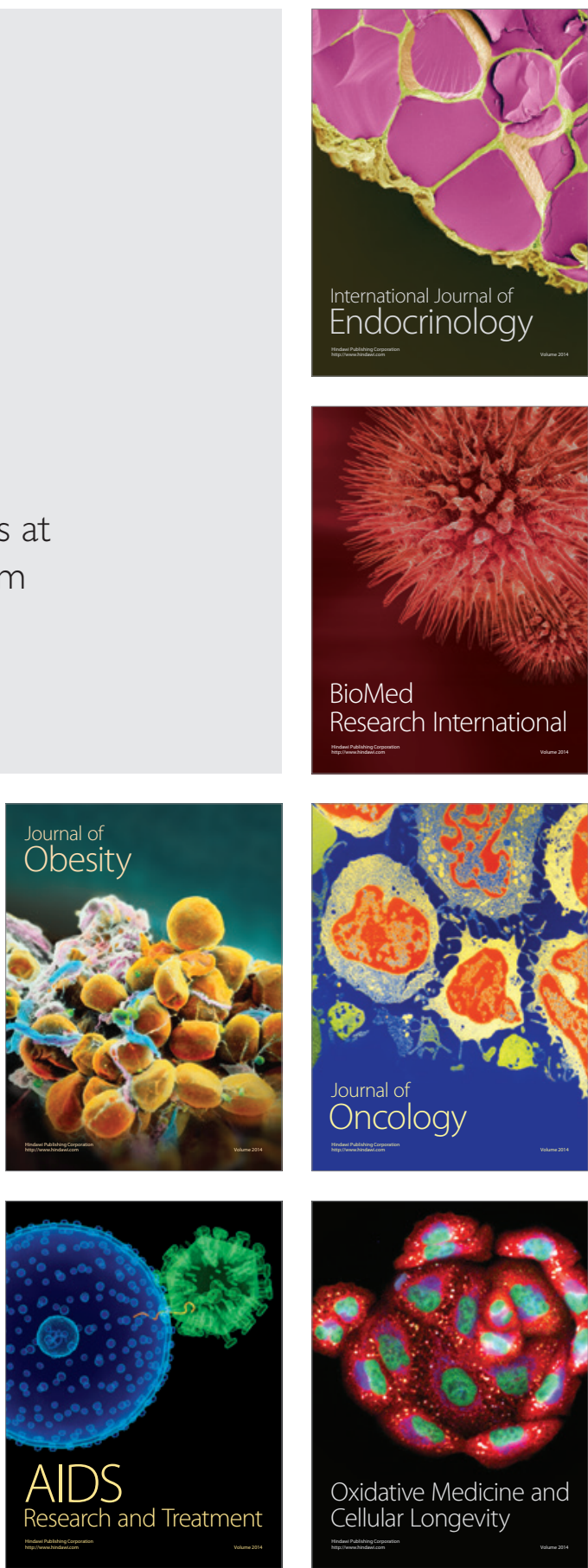